\title{
O valor formativo das comunidades de prática na construção da identidade profissional
}

\author{
Margarida Alvesi, Paula Queirósii \& Paula Batistaiii \\ Universidade do Porto, Portugal
}

Resumo

A formação de professores tem experienciado alterações substantivas no que diz respeito à sua concetualização e organização. Atendendo ao facto de a aprendizagem ser situada social e culturalmente, este texto de reflexão concetual procurou reequacionar o valor formativo das Comunidades de Prática para a construção da identidade profissional de futuros professores em contexto de estágio, bem como identificar os constrangimentos que pode encerrar. Da reflexão sobreveio o potencial das Comunidades de Prática no processo de construção da identidade profissional dos estagiários, que aqui encontram um espaço de partilha e de reflexão que lhes permite construir e reconstruir conceções, pelo assumir gradual de papéis potencialmente emancipatórios. Já as limitações incorrem das relações estabelecidas no seio das Comunidades de Prática quando estas se estruturam com base numa hierarquia excessiva e unidirecional.

Palavras-chave

Comunidade de Prática; Identidade profissional; Estágio; Estudantes estagiários

\section{Introdução}

Nos anos 90 do século XX, as alterações concetuais e estruturais na formação de professores resultaram no estabelecimento de parcerias entre as 
escolas e as instituições de formação de professores (van Velzen, Bezzina, \& Lorist, 2009). Estes consórcios, marcados por sinergias distintas, originaram grupos de trabalho com um mesmo propósito, podendo ser entendidos como Comunidades de Prática $\left(\mathrm{CoP}^{1}\right)$, sustentados na noção de que a aprendizagem é, inerentemente, situada. De facto, o reconhecimento, pelas instituições de formação, da necessidade de a aprendizagem para ser professor ser o mais situada possível é amplamente veiculado pela literatura, sendo que são diversos os estudos nesta área (Batista \& Pereira, 2014; Cuddapah \& Clayton, 2011; Edmonds-Cady \& Sosulski, 2012; Harris, 2011; Iza \& Neto, 2015; Skerrett \& Williamson, 2015). Neste entendimento, Queirós (2014) refere que é na escola, nos seus diálogos e rotinas, que se aprende a ser professor. É, portanto, notório que a formação de professores deve ser contextualizada, estabelecendo um fluxo recíproco entre as instituições de formação de professores e as escolas. Orsmond e Merry (2017) aludem ao facto de a aprendizagem não ser simplesmente o resultado de um determinado currículo, mas sim o resultado da participação em determinada prática, que no caso da formação de professores deve ocorrer nas escolas, no seio dos grupos profissionais.

O estágio, etapa essencial no contexto dos programas de formação de professores (Fletcher \& Kosnik, 2016; Smit, Fritz, \& Mabalane, 2010) e articulador de diferentes conhecimentos (Iza \& Neto, 2015), deve acontecer em grupo. Neste sentido, o estágio deve possuir um carácter de trabalho coletivo em que tanto os estudantes estagiários $(\mathrm{EE})$, como os orientadores da faculdade e da escola desempenham um papel fundamental na coconstrução de novo conhecimento (Kim Chuan, Wong, Choy, \& Tan, 2009).

Partindo deste entendimento, as CoP em contexto de estágio permitem aos EE integrarem-se em grupos que possuem um objetivo comum, no qual adotam novos papéis, negoceiam dilemas e vão aprendendo os elementos que perfazem a profissão docente. Esta aprendizagem decorre da imersão na CoP e contribui, simultaneamente, para a construção de uma Identidade Profissional (IP). A IP pode ser entendida como o tipo de pessoa que se é em determinado contexto (Gee, 2000), sendo este um processo que decorre ao longo de toda a vida, que é instável, dinâmico, permeável e é fortemente influenciado pelas experiências passadas, crenças e valores (Beltman, Glass, Dinham, Chalk, \& Nguyen, 2015; Moss \& Pittaway, 2013; 
Pillen, Beijaard, \& den Brok, 2013; Trent \& Shroff, 2013). Assim sendo, pode assumir-se a IP como um conceito simultaneamente biográfico e relacional, em que os aspetos pessoais e contextuais interagem de forma recíproca no sentido de a moldar (Beltman et al., 2015; Ruohotie-Lyhty \& Moate, 2016). É, então, pertinente que no estágio se considere que a construção da IP ocorre em comunidade (Forbes \& Davis, 2012; Gu, 2013; Williams, 2010), na qual os membros mais experientes permitem o acesso dos EE à mesma, ao conhecimento e à prática que os caracteriza. Orsmond e Merry (2017) veiculam esta ideia referindo que aprender a ser professor é um processo que tem lugar, sobretudo, no seio de uma CoP, sendo que as instituições formadoras de professores necessitam de colocar o seu enfoque nesta prerrogativa.

Face ao exposto, o interesse das CoP enquanto espaço de aprendizagem parece ser inestimável. Todavia, este conceito não tem sido devidamente explorado enquanto ferramenta formativa por excelência e pouco se tem investigado acerca da forma como as CoP podem auxiliar os futuros professores a construírem a sua IP (O'Brien \& Bates, 2015). Desta forma, o propósito central desta reflexão é, a partir da exploração do conceito de CoP, indagar acerca do seu valor formativo na construção da IP do EE no contexto da formação de professores, bem como dos constrangimentos que pode encerrar. Adicionalmente, o seu estudo e incorporação na formação de professores advém da necessidade de reconfigurar a formação de professores face às alterações que esta tem vindo a sofrer, em que se pretende uma coconstrução da prática, recorrendo à supervisão colaborativa e interativa e, portanto, menos modeladora e prescritiva.

\section{Afinal, o que é uma Comunidade de Prática?}

O conceito de CoP é um conceito que, devido ao seu imenso potencial no foro da aprendizagem, é extremamente abrangente. Importa, por isso, que a sua definição seja clara para que possamos, então, perscrutar as suas características e aplicações como um todo.

O termo CoP foi utilizado pela primeira vez por Lave e Wenger, no ano de 1991. Estes autores basearam-se na observação do processo de ensinoaprendizagem de cinco grupos de aprendizes: as parteiras de Yucatec (estado 
do México), alfaiates de Vai e Gola (tribos da Libéria), contramestres navais, talhantes e alcoólicos anónimos. Durante o processo descrito pelos autores, cada comunidade recebe novos membros que irão ser ensinados pelos membros mais antigos e proficientes face ao domínio da prática que os caracteriza. Outros trabalhos seminais se seguiram, com Wenger em 1998 e, posteriormente, com Wenger, McDermott e Snyder, em 2002. Atendendo ao facto de este ser um conceito em constante transformação (Li et al., 2009) e, por conseguinte, permeável a novas realidades e contextos, bem como à coexistência de diferentes definições, esta reflexão estruturou-se em torno do seguinte conceito de CoP:

Communities of practice are groups of people who share a concern, a set of problems, or a passion about a topic, and who deepen their knowledge and expertise in this area by interacting on an ongoing basis. (Wenger, McDermott, \& Snyder, 2002, p. 4)

Kirk e Macdonald (1998) referem que uma CoP consiste numa qualquer coletividade ou grupo que em conjunto contribui para as práticas partilhadas ou públicas em contextos específicos do quotidiano. Wenger et al. (2002) referem que existem inúmeras CoP e que todos pertencemos a diferentes CoP (trabalho, escola, atividades extracurriculares, entre outras), sem que para isso seja necessário trabalhar com os restantes membros de forma sistemática. Atualmente, a ligação entre os membros de uma CoP baseia-se, sobretudo, nos interesses e objetivos em comum, e não tanto na localização geográfica (Li et al., 2009). Por outro lado, nem todas as CoP têm nome ou são imediata e indubitavelmente reconhecidas; por vezes, somos apenas participantes ocasionais em algumas CoP, que podem ou não ser estruturadas propositadamente (Wenger et al., 2002).

Apesar da importância atribuída a este constructo, pode, por vezes, tornar-se difícil a compreensão da extensão do mesmo, ou seja, que grupos afinal se podem vislumbrar como CoP? Wenger et al. (2002) alertam para o facto de nem todas as comunidades serem CoP, já que estas se referem a um tipo de estrutura social específica com um objetivo também ele específico. Li et al. (2009) advogam, ainda, que rotular um grupo de pessoas como uma comunidade de aprendizagem não significa que ela funcione como tal. Neste sentido, as CoP devem surgir autonomamente de forma a evitar alguns dos constrangimentos relacionados com a sua constituição em resultado de forças 
exteriores, criando assim um certo sentido de artificialidade. Desta forma, uma CoP não é sinónimo de grupo ou equipa (Wenger, 2008). De acordo com Wenger (2008), as CoP materializam-se através de três dimensões: envolvimento mútuo (mutual engagement), empreendimento conjunto (joint enterprise) e, finalmente, o reportório partilhado (shared repertoire). O autor alega que a prática não existe em abstrato, logo ela apenas pode existir quando os participantes se envolvem em ações, num processo de negociação mútua de significados. Neste concreto, pode entender-se o envolvimento mútuo enquanto forma como os membros de uma determinada CoP interagem entre si; o empreendimento conjunto refere-se aos objetivos comuns; e, por fim, o reportório partilhado refere-se ao desenvolvimento de recursos comuns (Garner \& Hill, 2017).

A estrutura básica de uma CoP não estaria completa sem se referir outros três elementos fundamentais: domínio (domain), comunidade (community) e prática (practice) (Wenger et al., 2002). As CoP trabalham com um domínio particular que procuram desenvolver em conjunto, têm uma comunidade bem definida e na qual a comunicação entre os diferentes membros é incentivada, e exercem um tipo específico de prática (Jones, Stephens, Branch-Mueller, \& de Groot, 2016; Tavakoli, 2015). No que diz respeito ao domínio, ele cria um sentimento de identidade comum que, quando bem definido, legitima a comunidade através da afirmação do seu propósito e valor aos seus membros. A comunidade é um elemento crucial, porquanto a aprendizagem está intimamente relacionada com o sentimento de pertença, bem como com um processo intelectual. Por conseguinte, fazer parte de uma CoP suscita nos seus membros um sentimento de segurança e de privilégio ( $\mathrm{Lu}, 2016$; Wenger et al., 2002). Finalmente, a prática refere-se ao conhecimento específico que a comunidade desenvolve, partilha e mantém e pode incluir ideias, informações, histórias, documentos, entre outros elementos. É através da manutenção da homeostasia destes elementos da estrutura básica de uma CoP que ela prospera e se assume como uma entidade de desenvolvimento e partilha de conhecimento por excelência.

Atendendo aos aspetos supracitados, pode, então, afirmar-se que as CoP são comunidades de partilha e de aprendizagem bastante complexas e que podem apresentar características tão diversas como serem pequenas ou grandes, durarem mais ou menos anos, os seus membros partilharem a 
mesma localização geográfica ou não, serem homogéneas ou heterogéneas, serem intra ou interdisciplinares, espontâneas ou intencionais, e, finalmente, serem perfeitamente institucionalizadas ou não serem reconhecidas, de todo, pelas instituições (Wenger et al., 2002).

Independentemente da posição de uma CoP neste largo espectro e das características que as CoP apresentam, importa que nenhuma delas seja tida como mais ou menos valiosa, sendo que todas elas contribuem para a construção e partilha de novo conhecimento da forma mais adequada ao contexto em que se inserem.

\section{Construção de uma Comunidade de Prática - Qual caminho a seguir?}

Uma CoP é uma entidade orgânica, permeável e que se desenvolve ao longo do tempo, podendo ou não modificar algumas das suas características. Exatamente por isso, este é um processo que encerra algumas fases, tanto iniciais, como o planeamento e o próprio espoletar da CoP, como avançadas, no que diz respeito ao desenvolvimento e manutenção da mesma.

Assim sendo, e de acordo com Wenger et al. (2002), o desenvolvimento de uma CoP passa por 5 estádios, a saber: potencial, coalescente, maturação, manutenção e transformação². Durante este processo, os níveis de energia e de visibilidade da CoP alteram-se e vão surgindo diferentes tensões. No primeiro estádio - potencial - um dos aspetos primordiais é encontrar traços comuns entre os diferentes membros da CoP para que eles se sintam parte constituinte da mesma - este é um momento de descoberta; no segundo estádio - coalescente - é crucial que existam diferentes atividades e momentos para que se criem laços, uma relação de respeito, confiança e uma maior consciência dos interesses e necessidades em comum - funciona como um período de 'incubação'; durante o estádio de maturação e após a CoP ter demonstrado a sua validade, existe uma maior interação entre os diferentes membros, que trabalham no sentido de desenvolver novo conhecimento e de suprir as suas lacunas - existe uma preocupação crescente com o foco e com a expansão da CoP e seus constituintes; no quarto estádio - manutenção - assiste-se a uma alternância de altos e baixos que ilustram a dificuldade de manter a CoP ativa, diligente, 
e, ainda, a relevância do seu domínio - dicotomia entre a abertura ou relutância a novas perspetivas; a transformação é o último estádio de desenvolvimento de uma CoP. A partir do momento em que os limites, domínio, instrumentos e práticas de uma CoP ficam cada vez mais bem definidos, pode verificar-se alguma tensão no que diz respeito à diluição ou estreitamento dos limites da CoP. Essa constante reinvenção, ou até um evento marcante, pode, em última instância, dar lugar ao seu término, e este fim deve ser visto de uma forma natural.

Com o intuito de potenciar a construção de um CoP, Probst e Borzillo (2008) elencaram algumas das razões que podem estar na origem do seu sucesso, sendo que as seguintes parecem ser as mais relevantes do ponto de vista da formação de professores: definir objetivos estratégicos, conceder à CoP fontes de conhecimento externas, promover o acesso a outras CoP, o líder da CoP deverá ter um carácter de guia e facilitador e ultrapassar os constrangimentos relacionados com a hierarquia. No que diz respeito às razões que eventualmente poderão originar o insucesso da CoP, os autores mencionam a falta de um grupo coeso, a falta de identificação com a CoP e, por último, um baixo nível de interação entre os membros. É importante ressalvar que a construção de uma CoP deve sempre adequar-se às características, necessidades e objetivos da comunidade em causa.

\section{Membros da Comunidade de Prática: Quem é quem?}

Os membros que constituem uma CoP são uma das forças motrizes que poderá ditar o seu sucesso ou fracasso, pelo que é da maior relevância que esta temática seja aprofundada.

Wenger et al. (2002) referem que é irrealista esperar que todos os membros participem na CoP da mesma forma, uma que vez que as motivações pelas quais o fazem são, naturalmente, distintas. A motivação que determinou a adesão a uma CoP nem sempre é a mesma que veicula a permanência dos seus membros e é esta variedade de motivações, de intensidade e frequência de participação que acabam por confluir para a criação de uma CoP equilibrada.

As CoP possuem um facilitador, detentor de uma posição de liderança, e que é, usualmente, responsável por organizar ações, por identificar os 
temas mais pertinentes a abordar e por ser o elemento agregador entre os restantes membros da CoP (Baker \& Beames, 2016). Não obstante, outros membros podem desempenhar uma função de liderança. Existe um grupo nuclear que participa ativamente nas mais diferentes formas de reunião e projetos da CoP, sendo que muitas vezes também assume posições de liderança e de auxílio ao facilitador, devendo ser tido em conta na sucessão do líder (Baker \& Beames, 2016; Wenger et al., 2002). Uma outra forma de pertencer a uma CoP é fazendo parte do grupo ativo, que participa nas atividades ocasionalmente mas não com a regularidade e intensidade do grupo nuclear (Wenger et al., 2002). A maioria dos membros da CoP ocupa uma posição periférica por inúmeras razões, como, por exemplo, não terem tempo suficiente para participar mais ativamente, por acharem não ter autoridade para o fazer ou porque entendem que os seus comentários não são apropriados. Segundo Baker e Beames (2016), pela observação das rotinas da $\mathrm{CoP}$, os membros que se encontram na periferia podem decidir aumentar o seu grau de intervenção e participação, devido ao valor que atribuem a essas práticas. Para lá destes três níveis de participação distinta, existem pessoas exteriores à CoP mas igualmente importantes.

Este fluxo constante entre os diferentes níveis de participação, entre periferia e centro da $\mathrm{CoP}$, processa-se através da participação legítima periférica $^{3}$, conceito sugerido por Lave e Wenger, em 1991, e que se baseia na premissa de que a aprendizagem é uma atividade situada em que os novos membros legitimam a sua participação numa CoP através do sucesso do seu trabalho, evoluindo progressivamente de uma participação periférica para uma participação plena na CoP. Assim sendo, os novos membros da CoP possuem mais do que uma posição de observação, já que a participação é uma forma de aprendizagem, assimilam e são assimilados pela CoP e, através da imersão crescente na CoP e das interações estabelecidas com os membros mais antigos da CoP, desenvolvem uma ideia do seu domínio, do tipo de conhecimentos envolvidos e das suas práticas, ou seja, progridem em direção à participação plena (Farnsworth, Kleanthous, \& Wenger-Trayner, 2016; Lave \& Wenger, 2011; Lu, 2016).

Falar da interação estabelecida entre os diferentes membros da CoP sem mencionar as relações de poder instituídas é uma tarefa árdua, uma vez que a noção de participação periférica legítima é um conceito envolto em 
complexidade e que, segundo Liu e Xu (2013), a trajetória de aprendizagem dos professores é fortemente moldada pelas múltiplas relações de poder estabelecidas numa CoP. Para Roberts (2006), o poder constitui-se como a capacidade de alcançar um desiderato, seja através do controlo, da força ou da influência. As relações de poder são, então, estabelecidas, implementadas e consolidadas através do discurso e do significado que o mesmo gera (Hardy \& Maguire, 2016). Nas palavras de Fiske, Dupree, Nicolas, e Swencionis (2016), as relações de poder, a capacidade de agenciamento e a própria ação estão interligadas. Todavia, os novos membros não se comportam apenas como recetáculos de nova informação; eles contribuem, igualmente, para o processo de aprendizagem e transformação da CoP (Wenger, 2008). É através desta interação, da pertença à $\mathrm{CoP}$ e, por conseguinte, do processo de aprendizagem, que tanto os novos membros como os membros mais antigos (re)constroem a sua identidade, uma vez que a comunidade influencia as suas perspetivas e crenças ( $L u, 2016)$, tratando-se de mais do que a mera acumulação de aprendizagens e informações, ou seja, pressupõe um processo de transformação (Wenger, 2008).

\section{Comunidades de Prática e construção da identidade - Que ligação?}

A pertença a uma CoP, a aprendizagem que dela advém e a construção da identidade são conceitos inextrincáveis, já que, segundo Wenger (2008), envolvem a transformação do participante num certo tipo de pessoa, tratando-se, portanto, de uma experiência de identidade (experience of identity). Assim sendo, desenvolvem-se novas identidades através da participação numa CoP, recorrendo à utilização do mesmo tipo de linguagem, comportamento, negociando e partilhando significados e aprofundando o conhecimento acerca do seu domínio e prática (Handley, Sturdy, Fincham, \& Clark, 2006; Lu, 2016; Sfard, 1998). Pode, então, afirmar-se que o conhecimento, a pertença social e a identidade se relacionam e influenciam mutuamente, dado que a aprendizagem em contexto de CoP é reificada através da partilha entre os diferentes membros (Garner \& Hill, 2017; Lave \& Wenger, 2011).

Wenger (2008) chama a atenção para um aspeto fundamental, referindo que a identidade de alguém é definida não só pelas práticas nas 
quais se envolve, mas também pelas práticas nas quais não se envolve, ou seja, a identidade é constituída não só por quem somos, mas, igualmente, por quem não somos. Desta forma, e de acordo com o mesmo autor, a construção da identidade consiste na negociação do significado atribuído à nossa participação e às nossas experiências na CoP. De facto, a identidade depende do seu contexto de definição e de negociação (Dubar, 2006; Gee, 2000; Kriner, Coffman, Adkisson, Putman, \& Monaghan, 2015).

Nas palavras de Wenger (2008), a identidade possui características intrínsecas, tais como: envolve participação e reificação, não se tratando, portanto, de apenas um rótulo ou categoria; é negociada e envolve um processo complexo de transformação constante; é social, porquanto se desenvolve através das vivências em comunidade, podendo estas ser mais ou menos familiares; constitui-se como um processo de aprendizagem que incorpora tanto o passado como o futuro para atribuir significado às vivências do presente; combina diferentes formas de pertença negociadas e equilibradas através dos limites da CoP; e, por fim, nem é estritamente local nem global, mas sim uma combinação dos dois em que negociamos localmente formas de pertença mais abrangentes.

No que diz respeito ao processo de construção da identidade, um processo contínuo de negociação e renegociação (Brady, 2015), devemos ter em consideração três conceitos: compromisso - envolvimento ativo no processo de negociação e atribuição de significado através do qual a identidade se desenvolve; imaginação - criar novas perceções acerca do mundo e de nós como resultado do processo de aprendizagem, discussão e interação; alinhamento - coordenar a energia, investimento e atividades de forma a que se integrem em estruturas mais amplas (Wenger, 2008).

Assim sendo, pode concluir-se que a (re)construção da identidade é um processo complexo, contínuo, com um cariz extremamente social e dependente do contexto em que ocorre, sendo que a imersão numa CoP permite aos seus membros desenvolverem um conjunto diversificado de conhecimentos e um sentimento de pertença à mesma (Gee, 2000; Wenger, 2008). 


\section{O valor formativo das Comunidades de Prática para a construção da Identidade Profissional em contexto de Estágio}

As CoP têm sido utilizadas amplamente no âmbito educacional, com diferentes intuitos e intervenientes. Bouchamma e Michaud (2011), tendo como objetivo adaptar um programa de avaliação de professores, estudaram uma CoP constituída por diretores de escolas, vice-diretores e diretores de departamento responsáveis pela supervisão de professores; Cuddapah e Clayton (2011) conduziram um estudo na procura de perceberem se as CoP se constituem como um bom apoio sobretudo no caso de professores que se encontram a iniciar a sua carreira. Por sua vez, Parker, Patton, Madden, e Sinclair (2010) examinaram um grupo de professores de educação física enquanto CoP, cujo objetivo era disseminar um currículo escolar, e Williams (2010), recorrendo ao conceito de CoP, tentou indagar acerca da (re)construção da IP de alguém que mudou de profissão. Mais especificamente, o conceito de CoP tem tido um enfoque especial em contexto de estágio. Exemplos disso são o estudo de $\mathrm{Gu}$ (2013), que acompanhou seis EE que se encontravam a estagiar em Hong-Kong e que se socorreu do conceito de CoP para perceber a construção da sua IP; o de Joseph e Heading (2010), que conduziram um estudo de caso para descrever como é que um EE vivenciou a sua experiência de ensino e passou de uma identidade de estudante para uma identidade de professor; Maynard (2001) explorou os processos envolvidos no aprender a ser professor durante o estágio na escola; Morton e Gray (2010) focaram-se na CoP criada pelos EE e pelo supervisor com o objetivo de discutir o planeamento de aula; e, finalmente, Skerrett e Williamson (2015) tentaram perceber como é que um EE lidou com as diferentes CoP nas quais esteve envolvido enquanto se tornava professor.

No caso do contexto português e lusófono, surgem outros estudos, como o de Cardoso, Batista, e Graça (2016), que revela a dinâmica estabelecida numa CoP na reconstrução da IP de três EE através do olhar da professora cooperante; o de Cunha, Batista, e Graça (2014), que examina as representações acerca da IP que os EE do ensino superior público português detêm, resultantes do processo de formação de professores de educação física em CoP; Flores e Day (2006), embora não foquem especificamente as 
CoP, enfatizam as culturas escolares colaborativas ao discorrerem acerca dos fatores que permitiram a 14 novos professores reconstruir a sua IP através da interação entre fatores pessoais, profissionais e contextuais; Fogaça e Halu (2017) procuraram investigar os efeitos que um programa de formação de professores pode ter tido na construção da IP de professores universitários usando como referencial teórico o constructo de CoP; e, por fim, Cyrino (2016), que investigou grupos de formação de professores que se constituíram como CoP.

O estágio é considerado um momento de aprendizagem por excelência, em que os EE procuram levar para a prática o que aprenderam (Kim Chuan et al., 2009). No estágio, os EE encontram-se imersos num contexto social diferente do habitual, assumindo uma posição de novos membros em busca de novo conhecimento, sendo o acesso a esse conhecimento permitido ou não pelos membros mais antigos e experientes. Face a este panorama, e uma vez que aprender não é uma atividade individual, mas sim dependente das práticas sociais e culturais (Dumitru \& Enăchescu, 2015; Farnsworth et al., 2016; Kriner et al., 2015), torna-se evidente a aplicabilidade e pertinência do conceito de CoP, inserido num processo formativo. No entendimento de Wenger et al. (2002), de uma maneira geral, pode considerar-se que o estágio decorre no seio de uma CoP, porquanto os EE, a faculdade, as escolas, os professores cooperantes e os restantes membros envolvidos trabalham em conjunto face a um objetivo comum, negoceiam significados, interagem de forma contínua, produzem e partilham novo conhecimento que, em última instância, levará à (re)construção da IP. Cuddapah e Clayton (2011) referem que as CoP são espaços essenciais para a integração e aprendizagem de professores iniciantes. No estudo que estes autores coordenam, o grupo de professores iniciantes que estudaram não se situava na periferia, mas, pelo contrário, esses professores eram percecionados como elementos valiosos para a melhoria da CoP, ou seja, mais do que se posicionarem apenas como recetores de conhecimento, os EE podem e devem ser vistos como capazes de contribuir para a melhoria das práticas, estabelecendo uma relação de reciprocidade com os elementos mais antigos da CoP.

$\mathrm{Na}$ tentativa de compreender o percurso e a experiência de um EE durante o estágio, Skerrett e Williamson (2015) chegaram a conclusões 
semelhantes, isto é, o mútuo reconhecimento no que diz respeito aos recursos e capacidades entre os EE e os restantes membros da CoP concorre para o fortalecimento da CoP e das suas práticas. No estudo supracitado, o EE referiu que o facto de a sua posição ter sido legitimada pelos membros mais antigos da CoP, ainda que de forma periférica e contrariamente aos papéis de não participação ou marginalização frequentemente atribuídos aos $E E$, foi um aspeto fundamental na sua formação e desenvolvimento enquanto professor, ou seja, no processo de configuração da sua IP como professor. É, então, através do mútuo reconhecimento entre os diferentes membros da CoP que os $E E$ conseguem aceder às práticas reais. $O$ estágio foi vivenciado de forma análoga pelos participantes do estudo desenvolvido por ten Dam e Blom (2006), no qual os EE relataram sentir-se parte integrante da CoP, desempenhando funções semelhantes às dos restantes professores, como por exemplo participar em visitas de estudo, escrever artigos para o jornal da escola e falar com os pais. Uma vez mais, o acesso e integração num contexto real, da escola, foi elencado como primordial para a construção da IP de professor.

Neste entendimento, Wenger (2008) e Gee (2000) referem que a construção da identidade está intimamente relacionada com o contexto em que nos encontramos e com o tipo de pessoa em que cada um se transforma através das interações, experiências e relações estabelecidas. Tornar-se professor não está somente relacionado com a aquisição de um conjunto de conhecimentos e competências, mas, sobretudo, com o desenvolvimento de uma identidade pessoal e profissional, que será desenvolvida e enriquecida quando em contacto com as práticas reais das escolas e com o quotidiano dos professores (Orsmond \& Merry, 2017; van Velzen et al., 2009). A este propósito, Sfard (1998) utiliza duas metáforas para caracterizar e explicar o processo através do qual se aprende a ser professor: a metáfora da aquisição e a metáfora da participação. A metáfora da aquisição remete a aprendizagem para a aquisição e acumulação de algo, uma perspetiva em que a mente humana era percecionada como um recipiente a ser completado com novos conceitos sem que estes fossem refletidos. Contrariamente, na metáfora da participação a aprendizagem tem lugar quando o indivíduo se torna membro de uma determinada comunidade e participa nas atividades que the são inerentes, atribuindo-Ihe significado. Convém, assim, salientar, que a natureza 
situacional da aprendizagem se manifesta através da coparticipação em diferentes contextos físicos e sociais, sendo, por isso, inerentemente contextual e interativa (Kerno, 2008). De igual forma, ten Dam e Blom (2006) referem que aprender a participar nas práticas sociais e culturais em contexto escolar é crucial para desenvolver a IP do professor.

Trent (2013), reportando as expectativas de EE relativas ao estágio, referiu que para eles se tratava de um momento para definir a sua IP. Já Ruohotie-Lyhty e Moate (2016) advogam que fazer parte de uma CoP permite aos EE vislumbrar mais facilmente a sua futura IP, pois a participação incentiva-os a desenvolverem a sua capacidade de agenciamento, aqui entendida como a capacidade de participação e a responsabilidade pelo seu processo de ensino-aprendizagem. Com efeito, Eteläpelto, Vähäsantanen, Hökkä, e Paloniemi (2013) mencionam que a agência, em contexto profissional, está presente quando se fazem escolhas e se exerce influência no sentido de provocar uma alteração no trabalho e na IP. As experiências vivenciadas durante o estágio têm, assim, o potencial de contribuir para transformar o EE no tipo de professor que ele quer ser (Sinner, 2010), sendo esta uma fase vital para o desenvolvimento e melhoria das suas capacidades (Naz, Abida, Munir, \& Saddiqi, 2010). Por sua vez, Maynard (2001) refere que os EE começam a desenvolver a sua IP através da apropriação do discurso dos seus orientadores da escola, ainda que de forma inconsciente e apesar de nem sempre partilharem, numa fase inicial, as mesmas conceções e metodologias. Os EE, ao serem inseridos numa CoP, são muitas vezes aceites pelos restantes membros devido à adoção do seu discurso e comportamento. Em última análise, esta aceitação permite-lhes negociar significados e entendimentos mais ricos e adequados às suas próprias metodologias e crenças. Os novos membros de uma CoP, neste caso os EE, necessitam de oportunidades para praticarem e se tornarem experientes, para assimilarem novo conhecimento e, ainda, para produzirem e atribuírem significado à vivência em comunidade através da sua prática (Harrison, Lawson, \& Wortley, 2005; Morton \& Gray, 2010).

No entanto, isto nem sempre acontece e, no caso de uma CoP não estar perfeitamente legitimada ou de as relações de poder entre os novos membros e os antigos impossibilitarem o acesso à CoP e à participação plena na mesma, os EE podem enfrentar sérias dificuldades aquando da definição 
da sua IP, competência e acesso à profissão (Harrison et al., 2005; Yuan \& Lee, 2015). De acordo com Skerrett e Williamson (2015), os EE, historicamente, sempre se posicionaram como participantes periféricos, o que poderá estar relacionado com o carácter passageiro do estágio, já que a integração dos EE enquanto professores numa escola (participação plena) não é um dos objetivos iniciais do estágio (Wenger, 2008).

Num estudo onde se acompanhou a construção da IP durante o estágio, Yuan e Lee (2015) revelam que inicialmente os EE foram rejeitados e se sentiam como meros tarefeiros e na periferia da $\mathrm{CoP}$, sendo que a impossibilidade de serem agentes ativos na construção do seu conhecimento e participantes na CoP originou um sentimento de incompletude, frustração e depressão que colocou em causa o processo de construção da IP. Estes sentimentos foram gradualmente ultrapassados à medida que o seu mérito foi sendo reconhecido e foram sendo integrados na CoP pelo orientador da escola.

Neste âmbito, Correa, Martínez-Arbelaiz, e Gutierrez (2014) mencionam que muitas vezes se assiste a uma desarmonia entre a IP que um EE entende que irá desenvolver durante o estágio e aquela que na verdade constrói. Com efeito, muitas vezes o EE acaba por ser subjugado às crenças fortemente instituídas na CoP. Os mesmos autores revelam que o facto de os EE se posicionarem na periferia, das suas opiniões não serem ouvidas e de possuírem uma capacidade de decisão reduzida no que concerne aos seus alunos, metodologias de ensino e ao quotidiano geral da escola suscita sentimentos negativos como o ressentimento, frustração e desinteresse pelo estágio, aliados à dificuldade em construírem a sua IP. Neste âmbito foi, ainda, aludida a necessidade de reprimir a questão emocional para agir em conformidade com a CoP e, por conseguinte, serem aceites na mesma. De acordo com Harrison et al. (2005), é por esta razão que o relacionamento dos EE com um outro significante ou facilitador, como o orientador da faculdade e da escola, é tão importante. Williams (2010) relata a experiência de uma EE durante o seu estágio, ficando evidente que a sua confiança aumentou devido ao apoio, incentivo e aceitação no seio da CoP por parte da orientadora da escola.

Não obstante, nem todos os EE se sentem amparados e estimulados pelos seus orientadores, tal como Johnston (2016) atesta ao apresentar o 
relato de um EE que refere ter sido afastado do processo de tomada de decisão, propagando o sentimento de desconforto e inadequação, quando as relações estabelecidas com os restantes membros da CoP não estavam bem consolidadas. De acordo com a perspetiva dos EE, Freidus (2002) identificou quatro metáforas acerca desta temática: orientador enquanto prospetor, dramaturgo, treinador e, finalmente, enquanto negociador. Devido à complexidade e às dificuldades que podem surgir aquando do estabelecimento de relações entre os $\mathrm{EE}$ e os restantes membros da CoP (Woodgate-Jones, 2012), os supervisores da faculdade detêm um papel indispensável para suavizar e dissipar os eventuais problemas práticos e emocionais que advêm do EP (Correa et al., 2014). Neste concreto, Alves, Queirós, e Batista (2014) referem que a integração num grupo que partilha objetivos, dilemas e desafios pode contribuir para a melhoria das práticas dos futuros professores.

Face ao exposto, os programas de formação de professores beneficiariam em serem organizados em CoP e não enquanto uma série de conteúdos isolados, compartimentados e estanques (Skerrett \& Williamson, 2015). Especificamente, no contexto do estágio, as CoP assumem-se como espaços privilegiados de partilha e construção de conhecimentos, em resultado das interações estabelecidas entre os diferentes elementos, que fomentam o desenvolvimento profissional dos orientadores e a construção da IP dos EE. Através da imersão numa CoP e das constantes interações que daí advêm, formais e informais, é possível enriquecer o estágio e o processo de construção da IP, encontrando soluções em espaços, porventura, não antes explorados.

\section{Comunidades de Prática - Que limitações?}

O potencial das CoP em processos de formação é amplamente reconhecido (Cross \& Ndofirepi, 2015; James, Busher, \& Suttill, 2016; Mak \& Pun, 2015); contudo, Kerno (2008) e Roberts (2006) alertam para o facto de ser necessário atender às suas limitações e elementos menos positivos.

Nas palavras de Wenger et al. (2002), é importante perceber que as CoP não são a solução para todos os problemas e que, inevitavelmente, podem estar na origem de alguns conflitos. Com efeito, as CoP podem limitar 
a inovação, podem circunscrever o acesso ao conhecimento, podem espelhar desajustes da sociedade, e mesmo aquilo que à primeira vista parece positivo, como uma boa relação entre os membros da CoP, pode tornar-se num aspeto negativo, uma vez que laços demasiadamente fortes podem tornar-se um obstáculo à entrada de novos membros na CoP, bem como à aceitação de feedback e novos pontos de vista.

Em contexto de estágio, as críticas apontadas às CoP referem-se, sobretudo, à hierarquia existente no seu seio e às inevitáveis relações de poder estabelecidas, bem como à falta de uma distinção perfeitamente inequívoca entre uma posição periférica e marginal face à CoP (Davies, 2005; Hall, 2017; Mitra, 2008). Uma hierarquia fortemente estabelecida no seio da CoP pode originar um processo de ensino-aprendizagem unidirecional, no qual a transmissão de conhecimento é apenas feita por parte dos membros mais antigos e os EE são vistos como recetáculos de informação, ou seja, a posição de poder dentro de uma CoP é valorada mediante o conhecimento que cada participante detém. Neste sentido, Roberts (2006) menciona que os participantes mais antigos, devido ao papel que desempenham na CoP, são detentores de mais poder nos processos de negociação de significado produção de novo conhecimento. Ancorado neste pressuposto, Hall (2017) refere que o facto de por vezes existirem tensões entre os diferentes membros da CoP ou obrigatoriedade de acomodação face à mesma instiga a que a partilha de ideias, discussões e reflexões não seja totalmente franca, constrangendo a construção das IP. Também Mitra (2008) alerta para esta situação, referindo que examinar e compreender as relações de poder é crucial para perceber de que forma é que as tensões vivenciadas podem reprimir a geração de novo conhecimento no seio de uma CoP.

As relações de poder são, de igual forma, visíveis no que diz respeito ao acesso concedido aos EE pelos elementos mais antigos e detentores de conhecimento, assim como às respetivas formas de participação numa CoP. Davies (2005) refere que não existe uma distinção clara entre a participação periférica e marginal, parecendo apenas que a alguns participantes Ihes é dada a escolher a forma de participação e outros são, simplesmente, marginalizados. O mesmo autor acrescenta que, no caso de o acesso à CoP não estar dependente da aprendizagem e do envolvimento nas suas práticas, mas apenas da aceitação daqueles que possuem poder e estatuto dentro da 
CoP, a situação de aprendizagem afasta-se do que é desejável e adequado.

Por sua vez, Hall (2017) refere que o grande perigo de uma CoP é possuir uma hierarquia excessivamente delimitada e unidirecional, plasmada em relações de poder desequilibradas e em que o acesso à mesma não é claro e inequívoco. Nestas circunstâncias, e uma vez que a probabilidade de transformação é extremamente limitada, todo o potencial da CoP na (re)construção da IP fica, certamente, colocado em causa, nomeadamente em contexto de estágio.

O espectro das CoP é extremamente vasto, pelo que são inúmeros os desafios que podem ter que ultrapassar, sendo que o mais importante é estarse atento à saúde externa e interna de cada CoP para que ela consiga atingir o seu potencial máximo. Nesta senda, é fundamental que as instituições de formação de professores, aquando da configuração das CoP em contexto de estágio, tenham em consideração estes constrangimentos e funcionem como balizadores do processo evitando as limitações aqui identificadas.

\section{Considerações finais}

O contributo e o valor formativo das CoP aquando da (re)construção da IP em contexto de estágio parece ser amplamente aceite. A (re)construção da IP acontece quando os EE constroem o seu percurso da periferia para 0 centro da CoP, assumindo o controlo da sua formação.

Podemos sintetizar este processo de construção da IP em contexto de estágio dizendo que os EE se constituem como os novos membros de uma comunidade profissional e se situam, inicialmente, na periferia (Wenger, 2008). Através das interações e relações estabelecidas com os membros mais antigos, é-lhes concedido acesso ao domínio da CoP, ao seu conhecimento e à sua prática. O processo de (re)construção da IP decorre, então, em relação a essa CoP, através do compromisso, da imaginação e do alinhamento. Logo, a construção da IP decorre quando os EE obtêm acesso à prática e aprendem gradualmente quais as expectativas, limites e diretrizes de se ser professor (Dotger \& Smith, 2009). As CoP devem, assim, ser espaços de reflexão, em que haja tempo para analisar, produzir, construir e reconstruir pensamentos e conceções, através do questionamento inquieto e implicado de todos os intervenientes. A renegação da doutrinação do conhecimento e a crescente 
autonomização do EE no seio da CoP, pelo assumir de diferentes papéis potencialmente emancipatórios, a combinação da reflexão, experimentação e diálogo conduzem a transformações pessoais e profissionais dos EE, pela modelação do seu conhecimento enquanto professores e da sua IP. Nesta ambiência, em que as relações de poder estão mais diluídas, os futuros professores e orientadores constroem novos conhecimentos no coletivo, comprometidos com a qualidade educativa e com a renovação das práticas profissionais, sem, contudo, prescindirem da sua individualidade.

Apesar de o conceito de CoP ser bastante relevante no contexto da formação de professores e, nomeadamente, da (re)construção da IP, ele encerra igualmente alguns constrangimentos que poderão colocar em causa o seu desenvolvimento profícuo e valor formativo efetivo, com especial destaque para a falta de reciprocidade na relação estabelecida entre EE e orientadores, relações de poder desequilibradas entre os diferentes membros da CoP e, por conseguinte, excessiva hierarquização (Howlett, Arthur, \& Ferreira, 2016).

Deste modo, e à semelhança de Skerrett e Williamson (2015), ten Dam e Blom (2006), Woodgate-Jones (2012) e Garner e Hill (2017), acredita-se que as entidades formadoras de professores beneficiariam em organizar o EP como uma CoP, desenvolvendo um ambiente em que a aprendizagem possa ter lugar e cujos benefícios não sejam deixados ao acaso, criando mais espaços de partilha e discussão e estreitando as relações estabelecidas entre os diferentes níveis de participação, assegurando, assim, a continuidade e constante evolução da mesma. Um conhecimento aprofundado acerca do funcionamento da CoP possibilitaria o desenvolvimento de estruturas e processos que melhor suportassem a aprendizagem (Khalil, Ardoin, \& Wojcik, 2017). Mais concretamente, se a construção da IP for entendida na perspetiva da CoP e enquanto um princípio organizador dos currículos de formação de professores, os contextos criados tenderão a promover e a enquadrar a negociação que é desejável ao processo de (re)construção das IP (Farnsworth et al., 2016). 


\section{Notas}

1 Do inglês Communities of Practice.

2 No primeiro estádio - potencial - a CoP encontra-se numa fase rudimentar em que atrai um grupo informal de pessoas que começam a interagir e que, muito provavelmente, constituirão o núcleo da CoP; no segundo estádio - coalescente podemos observar um pico de energia e visibilidade da CoP; terceiro estádio maturação - há uma mudança de paradigma no sentido de clarificar o objetivo principal da CoP, o seu foco, bem como os seus limites; quarto estádio manutenção - manutenção do desenvolvimento da CoP relativamente à sua prática, aos seus membros, tecnologia e relações; quinto estádio - transformação - tensão no que diz respeito à diluição ou estreitamento dos limites da CoP.

3 "Legitimate peripheral participation provides a way to speak about the relations between newcomers and old-timers, and about activities, identities, artifacts, and communities of knowledge and practice. It concerns the process by which newcomers become part of a community of practice" (Lave \& Wenger, 2011, p. 29).

\section{Referências}

Alves, M., Queirós, P., \& Batista, P. (2014). Os processo de agenciamento e de estrutura no contexto do estágio profissional: A voz dos estudantes estagiários. In P. Batista, P. Queirós, \& A. Graça (Eds.), O estágio profissional da (re)construção da identidade profissional em Educação Física (pp. 209-240). Porto: FADEUP.

Baker, A., \& Beames, S. (2016). Good CoP: What makes a community of practice successful? Journal of Learning Design, 9(1), 72-79.

Batista, P., \& Pereira, A. L. (2014). Uma reflexão acerca da formação superior de profissionais de Educação Física: Da competência à conquista de uma identidade profissional. In I. Mesquita \& J. Bento (Eds.), Professor de Educação Física: Fundar e dignificar a profissão (pp. 75-101). Porto: FADEUP.

Beltman, S., Glass, C., Dinham, J., Chalk, B., \& Nguyen, B. (2015). Drawing identity: Beginning pre-service teachers' professional identities. Issues in Educational Research, 25(3), 225-245.

Bouchamma, Y., \& Michaud, C. (2011). Communities of practice with teaching supervisors: A discussion of community members' experiences. Journal of Educational Change, 12(4), 403-420.

Brady, J. (2015). Dialect, power and politics: Standard English and adolescent identities. Literacy, 49(3), 149-157. doi:10.1111/lit.12058

Cardoso, I., Batista, P., \& Graça, A. (2016). Narrativas acerca da formação de professores de Educação Física em contexto de prática supervisionada. Sociologia: Revista da Faculdade de Letras da Universidade do Porto, 32, 125145. doi:10.21747/0872-3419/soc32a6

Correa, J. M., Martínez-Arbelaiz, A., \& Gutierrez, L. P. (2014). Between the real school and the ideal school: Another step in building a teaching identity. Educational Review, 66(4), 447-464. doi:10.1080/00131911.2013.800956 
Cross, M., \& Ndofirepi, E. (2015). On becoming and remaining a teacher: Rethinking strategies for developing teacher professional identity in South Africa. Research Papers in Education, 30(1), 95-113.

Cuddapah, J. L., \& Clayton, C. D. (2011). Using Wenger's communities of practice to explore a new teacher cohort. Journal of Teacher Education, 62(1), 62-75.

Cunha, M., Batista, P., \& Graça, A. (2014). Um olhar sobre o estágio em Educação Física: Representações de estagiários do ensino superior público português. In P. Batista, P. Queirós, \& A. Graça (Eds.), O estágio profissional na (re)construção da identidade profissional em Educação Física (pp. 143-180). Porto: FADEUP.

Cyrino, M. C. C. T. (2016). Mathematics teachers' professional identity development in communities of practice: Reifications of proportional reasoning teaching. Bolema: Boletim de Educação Matemática, 30(54), 165-187. doi:10.1590/1980$4415 \mathrm{v} 30 \mathrm{n} 54 \mathrm{a} 08$

Davies, B. (2005). Communities of practice: Legitimacy not choice. Journal of Sociolinguistics, 9(4), 557-581. doi:10.1111/j.1360-6441.2005.00306.x

Dotger, B. H., \& Smith, M. J. (2009). "Where's the line?" - Negotiating simulated experiences to define teacher identity. New Educator, 5(2), 161-180.

Dubar, C. (2006). A crise das identidades. A interpretação de uma mutação. Porto: Edições Afrontamento.

Dumitru, D., \& Enăchescu, V. (2015). Communities of practice as a mean for decentralization. Procedia - Social and Behavioral Sciences, 187, 752-756. doi:10.1016/j.sbspro.2015.03.158

Edmonds-Cady, C., \& Sosulski, M. R. (2012). Applications of situated learning to foster communities of practice. Journal of Social Work Education, 1, 45-64.

Eteläpelto, A., Vähäsantanen, K., Hökkä, P., \& Paloniemi, S. (2013). Review: What is agency? Conceptualizing professional agency at work. Educational Research Review, 10, 45-65. doi:10.1016/j.edurev.2013.05.001

Farnsworth, V., Kleanthous, I., \& Wenger-Trayner, E. (2016). Communities of practice as a social theory of learning: A conversation with Etienne Wenger. British Journal of Educational Studies, 64(2), 139-160.

Fiske, S. T., Dupree, C. H., Nicolas, G., \& Swencionis, J. K. (2016). Status, power, and intergroup relations: The personal is the societal. Current Opinion in Psychology, 11, 44-48. doi:10.1016/j.copsyc.2016.05.012

Fletcher, T., \& Kosnik, C. (2016). Pre-service primary teachers negotiating Physical Education identities during the practicum. Education 3-13, 44(5), 556-565.

Flores, M. A., \& Day, C. (2006). Contexts which shape and reshape new teachers' identities: A multi-perspective study. Teaching and Teacher Education, 22, 219232. doi:10.1016/j.tate.2005.09.002

Fogaça, F. C., \& Halu, R. C. (2017). Comunidades de prática e construção identitária de formadores de professores em um programa de formação continuada. Revista Brasileira de Linguística Aplicada, 17(3), 427-454. doi:10.1590/19846398201711522 
Forbes, C. T., \& Davis, E. A. (2012). Operationalizing identity in action: A comparative study of direct versus probabilistic measures of curricular role identity for inquirybased science teaching. International Journal of Science and Mathematics Education, 10(2), 267-292. doi:10.1007/s10763-011-9292-1

Freidus, H. (2002). Teacher education faculty as supervisors/advisors/facilitators: Playing multiple roles in the construction of field work experiences. Teacher Education Quarterly, 29(2), 65-76.

Garner, P., \& Hill, D. M. (2017). Cultivating a community of practice to enable coach development in Alpine ski coaches. International Sport Coaching Journal, 4(1), 63-75.

Gee, J. P. (2000). Identity as an analytic lens for research in education. Review of Research in Education, 25, 99-125.

Gu, M. M. (2013). From pre-service to in-service teachers: A longitudinal investigation of the professional development of English language teachers in secondary schools. Educational Studies, 39(5), 503-521. doi:10.1080/03055698.2013. 801769

Hall, V. J. (2017). Exploring teacher-student interactions: Communities of practice, ecological learning systems - Or something else? Journal of Further and Higher Education, 41(2), 120-132. doi:10.1080/0309877X.2015.1070395

Handley, K., Sturdy, A., Fincham, R., \& Clark, T. (2006). Within and beyond communities of practice: Making sense of learning through participation, identity and practice. Journal of Management Studies, 43(3), 641-653. doi:10.1111/j.14676486.2006.00605.x

Hardy, C., \& Maguire, S. (2016). Organizing risk: Discourse, power, and "riskification". Academy of Management Review, 41(1), 80-108. doi:10.5465/amr.2013.0106

Harris, R. (2011). Learning how to be a teacher - Lessons the government needs to learn. Prospero, 17(3), 28-33.

Harrison, J. K., Lawson, T., \& Wortley, A. (2005). Mentoring the beginning teacher: Developing professional autonomy through critical reflection on practice. Reflective Practice, 6(3), 419-441. doi:10.1080/14623940500220277

Howlett, C., Arthur, J. M., \& Ferreira, J. A. (2016). Good CoPs and bad CoPs: Facilitating reform in first-year assessment via a community of practice. Higher Education Research and Development, 35(4), 741-754.

Iza, D. F. V., \& Neto, S. D. S. (2015). Por uma revolução na prática de ensino: O estágio curricular supervisionado. Curutiba, Brasil: CRV.

James, N., Busher, H., \& Suttill, B. (2016). 'We all know why we're here': Learning as a community of practice on access to HE courses. Journal of Further \& Higher Education, 40(6), 765-779.

Johnston, D. H. (2016). 'Sitting alone in the staffroom contemplating my future': Communities of practice, legitimate peripheral participation and student teachers' experiences of problematic school placements as guests. Cambridge Journal of Education, 46(4), 533-551. doi:10.1080/0305764X.2015.1069793

Jones, K. M. L., Stephens, M., Branch-Mueller, J., \& de Groot, J. (2016). Community of practice or affinity space: A case study of a professional development MOOC. Education for Information, 32(1), 101-119. 
Joseph, D., \& Heading, M. (2010). Putting theory into practice: Moving from student identity to teacher identity. Australian Journal of Teacher Education, 35(3), 7587.

Kerno, J. S. J. (2008). Limitations of communities of practice. Journal of Leadership \& Organizational Studies, 15(1), 69-78.

Khalil, K., Ardoin, N. M., \& Wojcik, D. (2017). Social learning within a community of practice: Investigating interactions about evaluation among zoo education professionals. Evaluation and Program Planning, 61, 45-54. doi:10.1016/j. evalprogplan.2016.12.001

Kim Chuan, G., Wong, A. F. L., Choy, D., \& Tan, J. P. I. (2009). Confidence levels after practicum experiences of student teachers in Singapore: An exploratory study. KEDI Journal of Educational Policy, 6(2), 121-140.

Kirk, D., \& Macdonald, D. (1998). Situated learning in Physical Education. Journal of Teaching in Physical Education, 17(3), 376-387.

Kriner, B. A., Coffman, K. A., Adkisson, A. C., Putman, P. G., \& Monaghan, C. H. (2015). From students to scholars: The transformative power of communities of practice. Adult Learning, 26(2), 73-80.

Lave, J., \& Wenger, E. (2011). Situated learning: Legitimate peripheral participation. Cambridge: Cambridge University Press.

Li, L. C., Grimshaw, J. M., Nielsen, C., Judd, M., Coyte, P. C., \& Graham, I. D. (2009). Evolution of Wenger's concept of community of practice. Implementation Science, 4, 1-8. doi:10.1186/1748-5908-4-11

Liu, Y., \& Xu, Y. (2013). The trajectory of learning in a teacher community of practice: A narrative inquiry of a language teacher's identity in the workplace. Research Papers in Education, 28(2), 176-195.

Lu, Y. L. (2016). Experiences in the workplace community and the influence of community experiences on ENP courses for nursing professionals. Nurse Education Today, 40, 39-44. doi:10.1016/j.nedt.2016.01.025

Mak, B., \& Pun, S.-H. (2015). Cultivating a teacher community of practice for sustainable professional development: Beyond planned efforts. Teachers \& Teaching, 21(1), 4-21. doi:10.1080/13540602.2014.928120

Maynard, T. (2001). The student teacher and the school community of practice: A consideration of 'learning as participation'. Cambridge Journal of Education, 31(1), 39-52.

Mitra, D. L. (2008). Balancing power in communities of practice: An examination of increasing student voice through school-based youth-adult partnerships. Journal of Educational Change, 9(3), 221-242. doi:10.1007/s10833-007-9061-7

Morton, T., \& Gray, J. (2010). Personal practical knowledge and identity in lesson planning conferences on a pre-service TESOL course. Language Teaching Research, 14(3), 297-317. doi:10.1177/1362168810365243

Moss, T., \& Pittaway, S. (2013). Student identity construction in online teacher education: A narrative life history approach. International Journal of Qualitative Studies in Education, 26(8), 1004-1018. doi:10.1080/09518398.2012.725139 
Naz, F., Abida, K., Munir, F., \& Saddiqi, A. F. (2010). Practicum: A need in teacher education. International Journal of Learning, 17(8), 443-459.

O'Brien, W., \& Bates, P. (2015). "Looking and feeling the part": Developing aviation students' professional identity through a community of practice. Teaching in Higher Education, 20(8), 821-832.

Orsmond, P., \& Merry, S. (2017). Tutors' assessment practices and students' situated learning in Higher Education: Chalk and cheese. Assessment and Evaluation in Higher Education, 42(2), 289-303. doi:10.1080/02602938.2015.1103366

Parker, M., Patton, K., Madden, M., \& Sinclair, C. (2010). From committee to community: The development and maintenance of a community of practice. Journal of Teaching in Physical Education, 29(4), 337-357.

Pillen, M., Beijaard, D., \& den Brok, P. (2013). Tensions in beginning teachers' professional identity development, accompanying feelings and coping strategies. European Journal of Teacher Education, 36(3), 240-260. doi:10.1080/02619768.2012.696192

Probst, G., \& Borzillo, S. (2008). Why communities of practice succeed and why they fail. European Management Journal, 26(5), 335-347. doi:10.1016/j.emj.2008.05.003

Queirós, P. (2014). Da formação à profissão: O lugar do estágio profissional. In P. Batista, P. Queirós, \& A. Graça (Eds.), O estágio profissional na (re)construção da identidade profissional em Educação Física (pp. 67-83). Porto: FADEUP.

Roberts, J. (2006). Limits to communities of practice. Journal of Management Studies, 43(3), 623-639. doi:10.1111/j.1467-6486.2006.00618.x

Ruohotie-Lyhty, M., \& Moate, J. (2016). Who and how? Preservice teachers as active agents developing professional identities. Teaching and Teacher Education, 55, 318-327. doi:http://dx.doi.org/10.1016/j.tate.2016.01.022

Sfard, A. (1998). On two metaphors for learning and the dangers of choosing just one. Educational Researcher, 27(2), 4-13.

Sinner, A. (2010). Negotiating spaces: The in-betweeness of becoming a teacher. AsiaPacific Journal of Teacher Education, 38(1), 23-37. doi:10.1080/13598660903 474171

Skerrett, A., \& Williamson, T. (2015). Reconceptualizing professional communities for preservice urban teachers. Urban Review, 47(4), 579-600. doi:10.1007/s11256015-0325-x

Smit, B., Fritz, E., \& Mabalane, V. (2010). A conversation of teachers: In search of professional identity. Australian Educational Researcher, 37(2), 93-106.

Tavakoli, P. (2015). Connecting research and practice in TESOL: A community of practice perspective. RELC Journal: A Journal of Language Teaching and Research, 46(1), 37-52.

Ten Dam, G. T. M., \& Blom, S. (2006). Learning through participation. The potential of school-based teacher education for developing a professional identity. Teaching and Teacher Education, 22, 647-660. doi:10.1016/j.tate.2006.03.003

Trent, J. (2013). From learner to teacher: Practice, language, and identity in a teaching practicum. Asia-Pacific Journal of Teacher Education, 41(4), 426-440. 
Trent, J., \& Shroff, R. H. (2013). Technology, identity, and community: The role of electronic teaching portfolios in becoming a teacher. Technology, Pedagogy and Education, 22(1), 3-20.

Van Velzen, C., Bezzina, C., \& Lorist, P. (2009). Partnerships between schools and teacher education institutes. In A. Swennen \& M. van der Klink (Eds.), Becoming a teacher educator - Theory and practice for teacher educators (pp. 59-74). Heerlen: Springer.

Wenger, E. (2008). Communities of practice. Learning, meaning and identity. Cambridge: Cambridge University Press.

Wenger, E., McDermott, R., \& Snyder, W. (2002). Cultivating communities of practice. Boston, Massachusetts: Harvard Business School Press.

Williams, J. (2010). Constructing a new professional identity: Career change into teaching. Teaching and Teacher Education, 26(3), 639-647. doi:10.1016/j.tate.2009.09.016

Woodgate-Jones, A. (2012). The student teacher and the school community of practice: An exploration of the contribution of the legitimate peripheral participant. Educational Review, 64(2), 145-160. doi:10.1080/00131911.2011.590180

Yuan, R., \& Lee, I. (2015). The cognitive, social and emotional processes of teacher identity construction in a pre-service teacher education programme. Research Papers in Education, 30(4), 469-491. 


\title{
THE EDUCATIONAL VALUE OF COMMUNITIES OF PRACTICE IN BUILDING PROFESSIONAL IDENTITY
}

\begin{abstract}
Teacher training has experienced substantive changes with respect to its composition and organization. Given that learning is socially and culturally situated, this conceptual reflection text aimed to re-evaluate the formative value of the Communities of Practice in the construction of the professional identity of future teachers within practicum, as well as recognize the constraints that Communities of Practice may entail. From the reflection, it can be emphasised the potential of the Communities of Practice in the process of building the professional identity of the preservice teachers, since they find a space of sharing and reflection that allows them to construct and reconstruct conceptions, by gradually taking on potentially emancipatory roles. Limitations, on the other hand, lie in the relations established within the Communities of Practice when they are structured on the basis of an excessive and unidirectional hierarchy.
\end{abstract}

Keywords

Community of Practice; Professional identity; Practicum; Preservice teachers

\section{EL VALOR FORMATIVO DE LAS COMUNIDADES DE PRÁCTICA EN LA CONSTRUCCIÓN DE LA IDENTIDAD PROFESIONAL}

\section{Resumen}

La formación de profesores ha experimentado cambios sustantivos en lo que se refiere a su conceptualización y organización. Dado que el aprendizaje se sitúa social y culturalmente, este texto de reflexión conceptual buscó reflexionar sobre el valor formativo de las Comunidades de Práctica para la construcción de la identidad profesional de futuros profesores en contexto de prácticas, así como identificar las limitaciones que pueden contener. De la 
reflexión se destaca el potencial de las Comunidades de Práctica en el proceso de construcción de la identidad profesional de los alumnos en prácticas, que aquí encuentran un espacio de compartición y de reflexión que les permite construir y reconstruir concepciones, por el asumir gradual de papeles potencialmente emancipatorios. Ya las limitaciones incurren en las relaciones establecidas en el seno de las Comunidades de Práctica cuando estas se estructuran sobre la base de una jerarquía excesiva y unidireccional.

\section{Palabras-clave}

Comunidades de Práctica; Identidad profesional; Prácticas; Alumnos en prácticas

Recebido em julho 2017 Aceite para publicação em setembro 2017

i Centro de Investigação, Formação, Inovação e Intervenção em Desporto (CIFI2D), Faculdade de Desporto, Universidade do Porto, Portugal.

ii Centro de Investigação, Formação, Inovação e Intervenção em Desporto (CIFI2D), Faculdade de Desporto, Universidade do Porto, Portugal.

iii Centro de Investigação, Formação, Inovação e Intervenção em Desporto (CIFI2D), Faculdade de Desporto, Universidade do Porto, Portugal.

Toda a correspondência relativa a este artigo deve ser enviada para: Margarida Alves, Faculdade de Desporto da Universidade do Porto, Rua Dr. Plácido Costa, 91, 4200-450 Porto. E-mail: margarida@fade.up.pt 